\title{
The generality of the levels of processing hypothesis: An application to memory for chess positions
}

\author{
DAVID M. LANE and LAUREN ROBERTSON \\ Rice University, Houston, Texas 77001
}

\begin{abstract}
The generality of the levels of processing approach to memory was tested by using chess positions rather than words as stimuli. Experiment 1 compared recall following semantic orienting instructions (find the best move and determine which side has the advantage), formal orienting instructions (determine the number of pieces on light squares and the number of pieces on dark squares), and intentional learning instructions using 19 novice chess players as subjects. Formal orienting instructions produced poorer recall than did either semantic orienting or intentional learning instructions, which yielded similar levels of retention. These results were replicated in Experiment 2 with 16 tournament chess players. Chess rating correlated with recall .82 under semantic orienting instructions but only -.15 under formal orienting instructions. It was concluded that the levels of processing framework has applicability outside the area of verbal learning.
\end{abstract}

The "levels of processing" view of human memory holds that the durability of a memory trace is determined chiefly by the level of cognitive analysis to which material is subjected (Craik \& Lockhart, 1972). "Shallow" analyses such as those which focus on physical aspects of the stimulus result in less permanent retention than "deeper" levels of processing which stress meaning.

Tests of this theory have typically involved verbal materials and comparisons of retention under intentional and incidental learning instructions. For example, Hyde and Jenkins (1969) found that incidental learning of words rated on a pleasantness-unpleasantness dimension (a semantic orienting task) was equal to that obtained under an intentional learning set. However, judging whether words contained either an " $\mathrm{e}$ " or a "g," a formal (nonsemantic) orienting task, resulted in very poor recall. Other studies have found consistently that formal orienting instructions produce poorer recall than either semantic or intentional learning instructions, which yield similar levels of retention (see Jenkins, 1974, for a review).

Some recent work, however, has forced a modification of the original "levels of processing" position. Craik and Tulving (1975) reported that words processed to the same semantic level could be differentiated by the amount of stimulus elaboration induced through the incidental instructions. Asked, for example, to indicate whether each stimulus word was or was not illustrative of a designated semantic category (e.g., furniture), subjects showed better retention for positive than for negative instances. Since either decision would require

The authors would like to thank Ronald Deike for his assistance in obtaining subjects. Requests for reprints should be sent to David M. Lane, Department of Psychology, Rice University, Houston, Texas 77001 semantic processing, but affirmation would involve more cognitive elaboration than rejection, it has been suggested that retention is enhanced by the richness and complexity of operations carried out on the stimulus (Craik, 1977).

The generality of the levels of processing theory would be enhanced if it could be shown to account for retention in settings other than those afforded by standard verbal learning paradigms. One task that has proved useful for the study of other human information processing phenomena is the game of chess (Chase \& Simon, 1973b). Although by no means completely "nonverbal," the game relies heavily upon the player's ability to conceptualize and store spatial patterns-a mode of organization the meaningfulness of which should be related to the player's level of skills or experience.

Several investigators have, in fact, shown that the ability to reconstruct briefly presented positions is strongly dependent on chess skill (Chamess, 1976; Chase \& Simon, 1973a; de Groot, 1965; Frey \& Adesman, 1976), but only for patterns that are plausible (Chase \& Simon, 1973a). This suggests an obvious analogy with meaningfulness of verbal material: Plausible positions are more meaningful than random ones, but only to the extent that the observer has learned their meaning; to the novice, all positions are relatively meaningless.

Early attempts to account for the superior recall of plausible positions by skilled players relied on the following hypothesis: During stimulus presentation, subjects recognize patterns of pieces on the board as corresponding to chunks stored in long-term memory; labels for these chunks are placed in short-term memory, with recall being constrained by the number of labels that can be held in short-term memory. According to 
this interpretation, labels represent larger chunks for skilled than for less skilled players, and it is this difference in chunk size, rather than a difference in the number of labels in short-term memory, that is primarily responsible for the relationship between chess skill and recall (Chase \& Simon, 1973a; Simon \& Gilmartin, 1973). Recent evidence has been inconsistent with the view that memory is constrained by the number of labels for chunks that can be held in short-term memory. Charness (1976) found that recall of chess positions is hampered only slightly by a 30 -sec interpolated task; he concluded that virtually all information about a chess position that is encoded is stored in long-term memory. Further, Frey and Adesman (1976) found that two chess positions can be remembered nearly as well as one. As these studies cast doubt on the interpretation of memory for chess positions presented by Chase and Simon, it appears reasonable to consider this phenomenon within the framework of levels of processing theory, particularly as modified to incorporate stimulus elaboration.

The present research, therefore, was designed to test the hypothesis that memory for chess positions is a function of the depth of processing and, particularly, of the richness of stimulus elaboration afforded by the combination of task and skill-level conditions. Two studies were carried out: The first, an adaptation of the Hyde and Jenkins (1969) paradigm, aimed at testing the levels of processing notion; the second, a correlational study, dealt with the skill-level hypothesis.

\section{EXPERIMENT 1}

This study attempted to extend the findings of Hyde and Jenkins (1969) to memory for chess positions. The semantic (meaningful) orienting task consisted of determining the best move as well as which side had the advantage. For the formal (nonmeaningful) orienting task, subjects were asked to count the number of pieces on light and on dark squares. We predicted that recall under intentional learning and semantic orienting instructions would be about equal, with recall under formal orienting instructions much lower.

\section{Method}

Subjects. The subjects were 19 undergraduate students at Rice University who played chess but had never competed in a chess tournament. Subjects received credit in a psychology class for their participation.

Materials. Two quiet (no exchanges under way) early middle game positions selected from "Chess Life and Review" were used. In each, there were a total of 28 pieces (14 white and 14 black) on the board. Described in Forsyth ${ }^{1}$ notation, the positions were as follows:

(1) r 2qk2r, 1pp2pp1, 1p2b2p, 2nnp3, 2B5, 3P1P2, PPP1NBPP, R2QK2R;

(2) r $2 \mathrm{q} 1 \mathrm{rlk}$, pp $2 \mathrm{bp} 1 \mathrm{p}, 2 \mathrm{npbp} 2,4 \mathrm{p} 3$, 4P3, 3QNN2, PPP2PPP, R1B2RK1.
The positions were presented on standard-size chess boards. The Guilford-Zimmerman Spatial Visualization Subtest, Form B (Guilford \& Zimmerman, 1953), was used as an interpolated task.

Procedure. All subjects viewed each of two chess positions for $20 \mathrm{sec}$, one position under intentional learning instructions and the other under either semantic or formal incidental learning instructions. After viewing both positions, subjects were given the spatial test as a distractor task. The test took $10 \mathrm{~min}$ and was used to insure that recall would not come from short-term memory. After completing the test, subjects were given an empty chess board and the 28 pieces associated with the first position they had viewed. They were instructed to reconstruct the first position they had seen, and were given $3 \mathrm{~min}$ to do so. Subjects were then presented with another empty board along with the 28 pieces associated with the second position. As before, $3 \mathrm{~min}$ were given to reconstruct the position.

In the intentional learning condition, subjects were instructed to study the position so as to be able to recall it at a later point in the experiment. Subjects in the semantic orienting condition were told that it was white to play and make his 13 th move, and that their task was to decide which side had the advantage and to determine the best move. White had a slight advantage in both positions. The formal task consisted of counting the number of pieces on black squares and the number of pieces on white squares. The order of presenting the intentional and incidental conditions was changed with each successive subject; 10 subjects received the intentional task first and 9 subjects received the incidental task first. The assignment of chess positions to conditions (intentional vs. incidental) and subjects to orienting instructions (semantic vs. formal) was determined randomly for each subject.

\section{Results and Discussion}

Recall scores were obtained by counting the number of correctly placed pieces. As can be seen in Table 1, retention in the formal orienting condition was lower than retention in either the semantic orienting condition $[F(1,17)=5.13, p=.0369]$ or the intentional condition $[F(1,9)=11.80, p=.0074]$; retention in the semantic orienting and intentional conditions was quite similar, and did not differ significantly $(\mathrm{F}<1)$. The conclusions of Hyde and Jenkins (1969) can therefore be generalized to memory for chess positions, indicating that the levels of processing framework of Craik and Lockhart (1972) is applicable to areas outside the one in which it was developed, verbal learning.

\section{EXPERIMENT 2}

Experiment 1 showed that, at least for novice chess players, memory for chess positions varies as a function

Table 1

Mean Recall in Experiments 1 and 2

\begin{tabular}{lrccc}
\hline & \multicolumn{2}{c}{ Experiment 1 } & \multicolumn{2}{c}{ Experiment 2 } \\
& Mean & SD & Mean & SD \\
\hline & \multicolumn{4}{c}{ Semantic } \\
Intentional & 14.44 & 4.59 & 20.00 & 4.14 \\
Incidental & 13.22 & 5.02 & 20.87 & 4.39 \\
& \multicolumn{4}{c}{ Formal Orienting Group } \\
Intentional & 14.80 & 6.43 & 18.25 & 5.09 \\
Incidental & 8.30 & 4.45 & 9.25 & 3.69 \\
\hline
\end{tabular}

Note-Maximum score $=28$. 
of depth of processing, with processing to the semantic level resulting in approximately the same level of recall as intentional leaming. In Experiment 2, we sought to replicate these findings using tournament chess players as subjects. We were also interested in exploring the relationship between chess ability and retention in the two incidental conditions. On the basis of Craik's (1977) theorizing, one would expect a positive relationship between chess skill and recall under semantic orienting instructions but little or no relationship between chess skill and recall under formal orienting instructions. In the former condition, the richer and more complex processing of the stronger players should enhance retention, whereas, in the latter, only superficial processing should occur regardless of the level of chess skill.

\section{Method}

Subjects. The subjects were 16 United States Chess Federation (USCF) rated players who either participated in or came to watch a local toumament in Houston, Texas. The ratings ranged from 1,150 to 2,050 , with a mean of $1,530.56$ and a standard deviation of 278.40 . Therefore, a large range of playing strengths was sampled. All subjects were clearly amateurs, however, as the cutoff for master is 2,200 and the cutoff for senior master is 2,400 . Subjects were paid $\$ 2$ for participating.

Procedure. There were a few differences between the procedures of Experiments 1 and 2. Due to the toumament players' greater familiarity with chess, positions were presented for 12 rather than $20 \mathrm{sec}$. The order of conditions (intentional first vs. incidental first), assignment of positions to conditions, and assignment of subjects to orienting instructions were counterbalanced rather than randomized.

\section{Results and Discussion}

The findings of Experiment 1 were replicated in Experiment 2. An inspection of Table 1 again reveals that retention in the formal orienting condition was lower than retention in either the semantic orienting condition $[F(1,14)=11.55, p=.0043]$ or the intentional condition $[F(1,7)=23.14, p=.0019]$, while retention in the semantic orienting and intentional conditions was almost identical $(F<1.0)$.

As can be seen in Figure 1, the relationship between USCF rating and recall was quite substantial for the semantic condition $(r=.82, p=.0120)$, while there was no statistically reliable relationship in the formal condition $(r=-.15)$. The difference between correlations was significant $(z=2.08, p=.0376)$.

The results of Experiment 2 indicate that the relationship between orienting instructions and retention is quite robust, holding for amateur as well as novice chess players. There is every reason to believe the same results would be found in an experiment with masterstrength players. The failure to find a relationship between chess skill and recall under formal orienting instructions parallels the finding by Chase and Simon (1973b) of no relationship between chess skill and memory for random positions; only when subjects are able to perceive the 64 squares and various pieces as a meaningful configuration does the player with the better understanding of chess show any superionity.

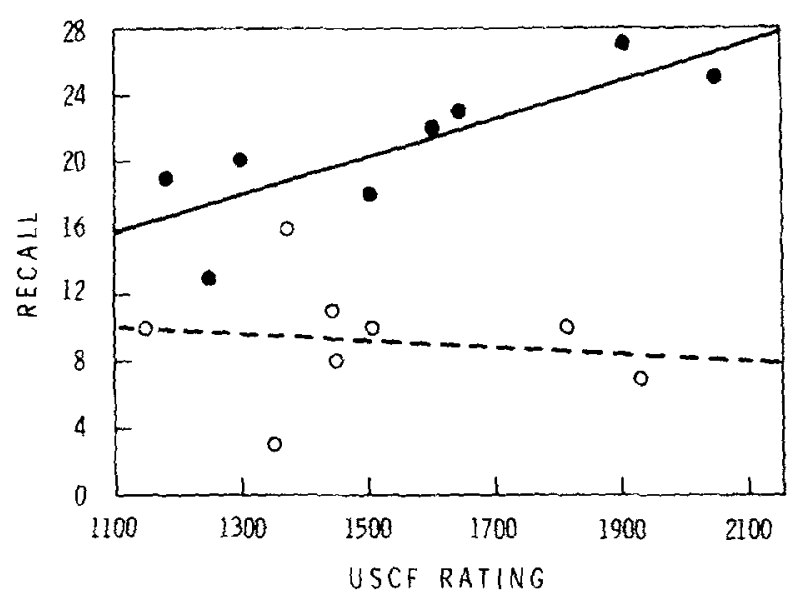

Figure 1. Regression of recall on chess rating for semantic orienting (closed circles) and formal orienting (open circles) conditions.

\section{GENERAL DISCUSSION}

These data, in conjunction with those of Chamess (1976) and Frey and Adesman (1976), provide strong evidence against Chase and Simon's (1973a) explanation of the relationship between playing strength and recall: A long-lasting representation of chess positions appears to be generated very quickly. The level of recall shown by the amateur players in the present experiments was quite remarkable. After viewing two chess positions for a total of $12 \mathrm{sec}$ each and performing a demanding spatial visualization test as an intervening task, subjects were able to correctly place over $70 \%$ of the pieces. Clearly, more than short-term memory is involved.

We believe that, in addition to being familiar with more patterns of chess pieces, stronger chess players are better than less able players at integrating familiar configurations into a coherent whole. Schermata for a chess position can be expected to play an important role in subsequent recall, just as the schemata of a short story appear to be important for both its summarization and recall (Rumelhart, 1977). Consistent with this view is the fact that the one position the masterstrength player studied by Chase and Simon (1973a) had trouble recalling was the one in which this player complained that he could not get the "sense" of the position. The semantic orienting task, but not the formal orienting task, used in the present experiments would seem to be conducive to the formation of schemata, and thus provide the stronger player an opportunity to utilize his superior understanding of the game.

We recently learned of a study by Goldin (in press), who also found better retention following a semantic than a formal orienting task; the present experiments and those of Goldin serve as independent replications of that phenomenon. The two most important findings of the present study, however, were not obtained by Goldin (in press). Our primary interest was in the comparison of intentional learning with incidental learning under semantic orienting instructions; Goldin did not 
include an intentional learning condition. Second, Goldin found only a marginal relationship between chess skill and retention. Moreover, no evidence that the relationship between chess skill and retention differs as a function of orienting instructions was obtained. The failure of Goldin (in press) to find the interaction between chess skill and orienting instructions evidenced in the present study may have resulted from (1) low power due to the fact that there were only three subjects in each of the three groups, (2) low power due to breaking up a continuous variable (chess rating) into discrete categories (see Cohen \& Cohen, 1975), or (3) a ceiling effect, as suggested by Goldin (in press), resulting from the use of recognition rather than recall as a measure of retention.

Lockhart (Note 1) argued that the data base for a theory of memory should be established by viewing memory as a by-product of cognitive-perceptual functioning. We believe the present results contribute to the establishrnent of such a data base by demonstrating that principles derived from the study of verbal learning can be applied successfully to very different stimuli, namely, chess positions. The next step is to consider what it is about cognitive-perceptual operations that influences memory. For the semantic orienting task used in the present experiments, one possibility is that it induces the subject to integrate the position into an organized and meaningful whole.

\section{REFERENCE NOTE}

1. Lockhart, R. S. The neglected component of levels of processing. Paper presented at the meeting of the Midwestern Psychological Association, Chicago, 1978.

\section{REFERENCES}

Charness, N. H. Memory for chess positions: Resistance to interference. Journal of Experimental Psychology: Human Learning and Memory, 1976, 2, 641-653.

Chase, W. G., \& Simon, H. A. Perception in chess. Cognitive Psychology, 1973, 4, 55-81. (a)
Chase, W. G., \& Simon, H. A. The mind's eye in chess. In W. G. Chase (Ed.), Visual information processing. New York: Academic Press, 1973. (b)

Conen, J., \& Cohen, P. Applied multiple regression/correlation analysis for the behavioral sciences. Hillsdale, N.J: Erlbaum, 1975.

CraIK, F. I. M. Depth of processing in recall and recognition. In S. Dornic (Ed.), Attention and performance VI. Hillsdale, N.J: Erlbaum, 1977.

Craik, F. I. M., \& Lockhart, R. S. Levels of processing: A framework for memory research. Journal of Verbal Learning and Verbal Behavior, 1972, 11, 671-684.

Craik, F. I. M., \& Tulving, E. Depth of processing and the retention of words in episodic memory. Journal of Experimental Psychology: General, 1975, 104, 268-294.

DE Groot, A. D. Thought and choice in chess. The Hague: Mouton, 1965.

Frey, P. W., \& Adesman, P. Recall memory for visually presented positions. Memory \& Cognition, 1976, 4, 541-547.

GoLdin, S. E. Effects of orienting tasks on recognition of chess positions. American Journal of Psychology, in press.

Guilford, J. P., \& Zimmerman, W. S. Guilford-Zimmerman aptitude survey. Orange, Calif: Sheriden Psychological Services, 1953.

Hyde, T. S., \& Jenkins, J. J. The differential effects of incidental tasks on the organization of recall of a list of highly associated words. Journal of Experimental Psychology, 1969, 82, 472-481.

JENKINS, J. J. Can we have a theory of meaningful memory? In R. L. Solso (Ed.), Theories in cognitive psychology: The Loyola symposium. Potomac, Md: Erlbaum, 1974.

RUMELHART, D. E. Understanding and summarizing brief stories. In D. LaBerge \& S. J. Samuels (Eds.), Basic processes in reading. Hillsdale, N.J: Erlbaum, 1977.

Simon, H. A., \& Gilmartin, K. A simulation of memory for chess positions. Cognitive Psychology, 1973, 5, 29-46.

\section{NOTE}

1. In this notation, capital letters represent white pieces and small letters black pieces. Each of the eight units, separated by commas, represents a rank (row), beginning with the eighth. The notation " $\mathrm{r} 2 \mathrm{qk} 2 \mathrm{r}$ " is short for "black rook, skip 2 squares, black queen, black king, skip 2 squares, black rook."

(Received for publication November 21, 1978; revision accepted March 19, 1979.) 